\title{
Prediction of the Elastic Properties of Single Walled Carbon Nanotube Reinforced Polymers: a Comparative Study of Several Micromechanical Models
}

\author{
Selmi Abdellatif and Hassis Hédi \\ Ecole Nationale d'Ingénieurs de Tunis, \\ Civil Engineering Laboratory \\ Tunisia
}

\section{Introduction}

The exceptional mechanical properties: Young's modulus, tensile strength, toughness, flexibility, etc. (Desprès et al., 1995) of carbon nanotubes (CNT) are due to their molecular structure consisting of tiny concentric graphene cylinders (Tijima, 1991). In order to determine the mechanical properties of this nano-structured material some theoretical and experimental methods were applied. Theoretical investigations yielded a widespread range of elastic property predictions, the predicted Young's modulus scatters in an interval of 0.55.5TPa (Lusti \& Gusev, 2004). The first experimental investigations of multi-walled nanotubes (MWNT) were based on the measurement of their intrinsic thermal vibration using a transmission electron microscope. Direct mechanical measurements of the Young's modulus were done using an atomic force microscope. Experimentally determined Young's modulus of single-walled carbon nanotubes (SWNT) scatter in a relatively large interval of 2.8 to 3.6TPa (Lourie \& Wagner, 1998). Their stiffness, strength, high aspect ratio, high Young's modulus and high flexibility, make CNT the best reinforcements of a polymer matrix.

Many parameters influence the overall effective properties of SWNT/Polymer composites, e.g. CNT structure, orientation, dispersion, diameter and matrix stiffness (Bai, 2006).

It was found (Lopez et al., 2005) by using a combined finite-element micromechanics approach that the degree of CNT waviness has a significant effect on the elastic properties of (NRP). The influence of the interaction at the CNT-polymer interface on the overall effective stiffness of NRP is investigated by linking molecular dynamics (MD) simulations with a micromechanical model (Odegard et al., 2003).

In this work, we develop and compare several micromechanical models. First, using a homogenization method based on energy equivalence, the nanotube's elastic properties are characterized.

Second, a direct finite element (FE) based numerical approach is used, which allows to model fully aligned, long and homogeneously dispersed SWNT in a SWNT/Polyimide composite.

Third, several mean-field homogenization schemes are investigated, namely: sequential, two-level (Mori-Tanaka/Mori-Tanaka): (M-T/M-T), two-step (M-T/M-T) and two-step (MT/Voigt) models. 
This is the main contribution of the paper. Indeed, while most papers dealing with the prediction of elastic properties of SWNT/Polymer composites study only one micromechanical model, in the present work we investigate 4 homogenization schemes and validate them against a fifth micromechanical approach: FE analysis of unit cells or representative volume elements (RVEs). Some predictions are also compared to experimental data or other micromechanical schemes.

The paper has the following outline. In section 2, the graphene sheet's elastic properties are characterized. In section 3, the investigated mean-field homogenization schemes are summarized. In section 4, several numerical predictions are presented. Conclusions are discussed in section 5 .

\section{Homogeneous elastic properties of graphene sheet}

A fundamental issue that needs to be solved in modelling macroscopic mechanical behaviour of nano-structured materials based on molecular structure is the large difference in length scales. The lengths associated to computational chemistry and solid mechanics are on the opposite ends of the length scale spectrum. Computational chemistry models predict molecular properties based on known quantum interaction and solid mechanics models predict the macroscopic mechanical behavior of materials idealized as continuous media based on known bulk material properties. To adress this issue, (Odegard et al., 2002) proposed a method for developing structure-property relationships of nano-structured materials. This method serves as a link between computational chemistry and solid mechanics by substituting discrete molecular structures with equivalent continuum models. It has been shown that this substitution may be accomplished by equating the molecular potential energy of the nano-structured material with the strain energies of the representative truss and continuum models. The technique developed by (Odegard et al., 2002) is applied here to the determination of the homogenous nanotube elastic properties. To reduce the computational time associated with modelling the graphene sheet, a representative volume element (RVE) for graphene was used. The RVE of the graphene's chemical structure has been substituted with equivalent truss and equivalent continuum models (Fig. 1).

\subsection{Chemical model}

The energy associated with the chemical structure which is formed by chemical bonds is given by:

$$
E^{\rho}+E^{\theta}=\sum_{m} \frac{k_{m}^{\rho}}{2}\left(\rho_{m}-P_{m}\right)^{2}+\sum_{m} \frac{k_{m}^{\theta}}{2}\left(\theta_{m}-\Theta_{m}\right)^{2}
$$

where the terms $P_{m}$ and $\Theta_{m}$ refer to the undeformed interatomic distance of bond $\mathrm{m}$ and the undeformed bond-angle $\mathrm{m}$, respectively.

The quantities $\rho_{m}$ and $\theta_{m}$ are the bond length and angle after stretching, respectively (Fig. 1). $k_{m}^{\rho}$ and $k_{m}^{\theta}$ are the force constants associated with the stretching and angle variation of bond and bond-angle $\mathrm{m}$, respectively. Using the parameters for the AMBER force field (Cornell et al., 1995), the force constants used are: 


$$
k_{m}^{\rho}=k^{\rho}=6.5210^{-7} \frac{n J}{\text { bond.nm } m^{2}}
$$

and

$$
k_{m}^{\theta}=k^{\theta}=6.5210^{-7} \frac{n J}{\text { angle.rad }}{ }^{2}
$$

\subsection{Truss model}

The truss model is formed by rods (Fig. 1) whose Young's moduli are given by (Odegard et al., 2002):

$$
E_{m}^{a}=\frac{R_{m}^{a} k_{m}^{\rho}}{A_{m}^{a}}, \quad E_{m}^{b}=\frac{12 k_{m}^{\theta}}{R_{m}^{b} A_{m}^{b}}
$$

where $\mathrm{m}$ is the rod number and a and $\mathrm{b}$ are types of rods. $R^{a}$ is the distance between two successive carbon atoms taken equal to $0.14 \mathrm{~nm}, \theta=2 \pi / 3$ and $R^{b}=\sqrt{3} R^{a}=0.2424 \mathrm{~nm}$. $A_{m}^{a}$ and $A_{m}^{b}$ are the cross sectional areas of the $\mathrm{m}^{\text {th }}$ rod of type a and $\mathrm{b}$, respectively (Odegard et al., 2002).

\subsection{Continuum model}

Knowing the characteristics of the two rod types, to determine the properties of the continuum model, the homogenization method based on energy equivalence is used. So it is assumed that the truss and continuum models are equivalent when the elastic strain energies stored in the two models are equal under identical displacement boundary conditions. The strain energies associated to the truss model for the two loading cases are calculated by the finite element method: CASTEM (CASTEM, 2000) program.

The area of the RVE is known and the homogeneous model-thickness $(0.34 \mathrm{~nm})$ is taken equal to the graphite interlayer distance (Krishnan et al., 1998). The mechanical properties of the truss elements have been determined as described above.

Knowing that the distance between two successive carbon atoms is the same, the nanotube structure is isotropic. So only two parameters (Young's modulus (E) and Poisson's ratio ( $v$ ) are needed to characterize the continuum model. Thus both the truss and continuum models were subjected to two sets of loading conditions. For each set, we get an equation which relates $\mathrm{E}$ and $v$.

For a pure shear strain in the $x_{1}-x_{2}$ plane (Fig. 2), the RVE is subjected to the following boundary conditions:

$$
\left\{\begin{array}{l}
u_{1}=0 \\
u_{2}=\gamma x_{1} \\
u_{3}=0
\end{array}\right.
$$

The continuum model associated energy is:

$$
U=\frac{V}{4} \frac{E}{(1+v)} \gamma^{2}
$$




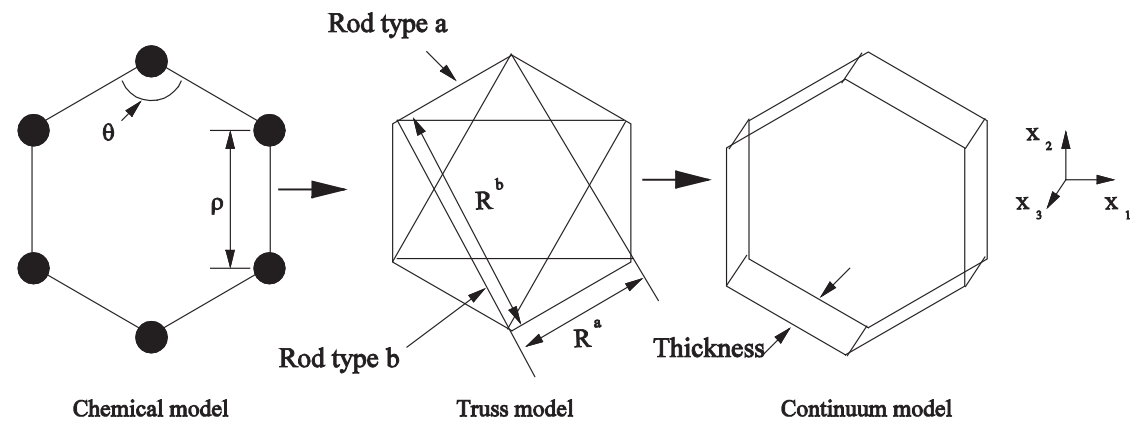

Fig. 1. Representative volume elements of the graphene sheet. Transition between chemical (bond distance $\rho$ and bond-angle $\theta$ ), truss (two rod types $R^{a}, R^{b}$ ) and continuum models, after (Odegard et al., 2002).

where $\mathrm{V}$ is the volume of the RVE which is given by:

$$
V=S t=\frac{3 \sqrt{3}\left(R^{a}\right)^{2} t}{2}
$$

Where $S$ is the hexagon's area and $t$ is the homogeneous model-thickness.

For a uniaxial deformation along the $x_{1}$ direction, the RVE is subjected to the following boundary conditions (Fig. 2):

$$
\left\{\begin{array}{l}
u_{1}=\gamma x_{1} \\
u_{2}=0 \\
u_{3}=0
\end{array}\right.
$$
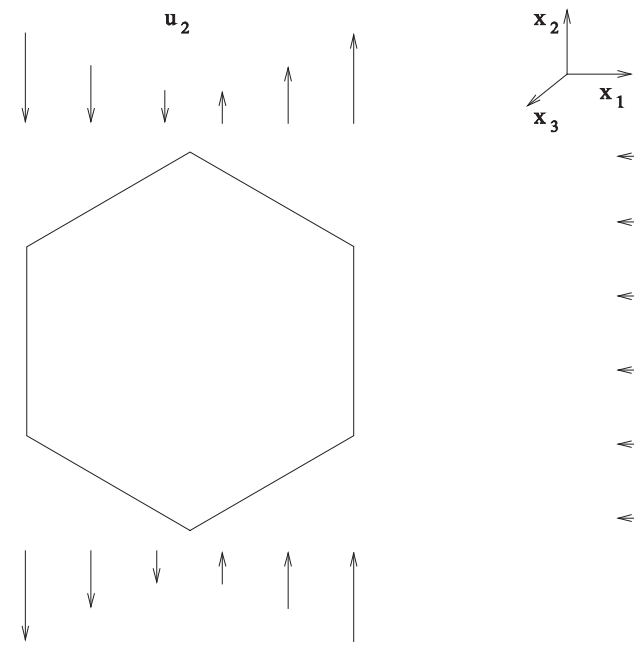

$u_{1}$

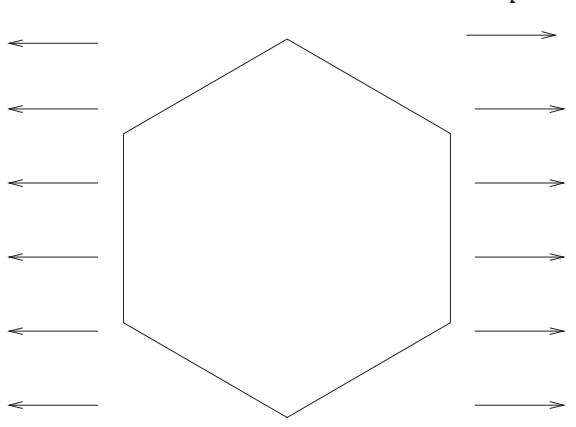

Fig. 2. Graphene RVE subjected to pure shear loading in the $\mathrm{x}_{1}-\mathrm{x}_{2}$ plane (left) and to extension in the $\mathrm{x}_{1}$ direction (right). 
The continuum model associated energy is:

$$
U=\frac{V E}{2(1+v)}\left[\frac{v}{(1-2 v)}+1\right] \gamma^{2}
$$

By equating the two strain energies for the truss and continuum model, we retrieve $\mathrm{E}$ and $v$ :

$$
\left\{\begin{array}{l}
E=2.52 \mathrm{TPa} \\
v=0.25
\end{array}\right.
$$

These elastic properties are entirely based on the strong intratube valence forces of the carbon-carbon bonds. The obtained Young's modulus value is retrieved close to the minimum value measured by (Lourie \& Wagner, 1998) with a micro-Raman spectroscopy (2.8-3.6TPa). It is also higher than the value measured by (Krishnan et al., 1998) with vibration method using a transmission electron microscope (1.45-2.25TPa).

\section{Overall elastic properties of SWNT composites}

SWNT composites can be seen as reinforced materials made up of three phases: polymer matrix, cylindrical cavities and carbon coatings. In this section, different ways to predict their overall elastic properties are explored: mean-field homogenization procedures, namely: the sequential method (Ben Hamida \& Dumontet, 2003), the two-step (Friebel et al., 2006) and the two-level (Friebel et al., 2006) procedures, and two dimensional unit-cell finite element calculations. The main ideas of these methods are summarised hereafter. Earmarks are underlined for each of them in the framework of CNT composites.

\subsection{The sequential method}

Many homogenization schemes are applicable only for low inclusion percentage, while in some cases it is needed to predict the influence of higher concentrations. Hence some homogenization schemes are improved with the iterative method (Ben Hamida \& Dumontet, 2003) which consists in the homogenization of the heterogeneous material in steps by adding the inclusions little by little. First, we have a homogeneous isotropic material, to it we add a volume $\delta V$ of isotropic inclusions (Fig. 3). The problem is solved by a given homogenization scheme. Then, this procedure is repeated iteratively by taking at step (i+1), the effective homogeneous material obtained at step (i). The end is detected when the inclusion percentage is reached.

For aligned long SWNT/Polymer composite, the considered RVE is formed by a unidirectional SWNT (void+continuum graphene) embedded in the matrix. In each homogenization step the problem is solved analytically for different elementary boundary conditions, and the overall macroscopic properties are thus computed.

\subsection{The two-step procedure}

The principle of the two-step procedure is depicted on Fig. 4 (bottom) for composites with aligned CNT. The coating phase (the carbon) is actually treated as a separate reinforcement phase. Carbon and void inclusions (both having the same shape and orientation) are thus 


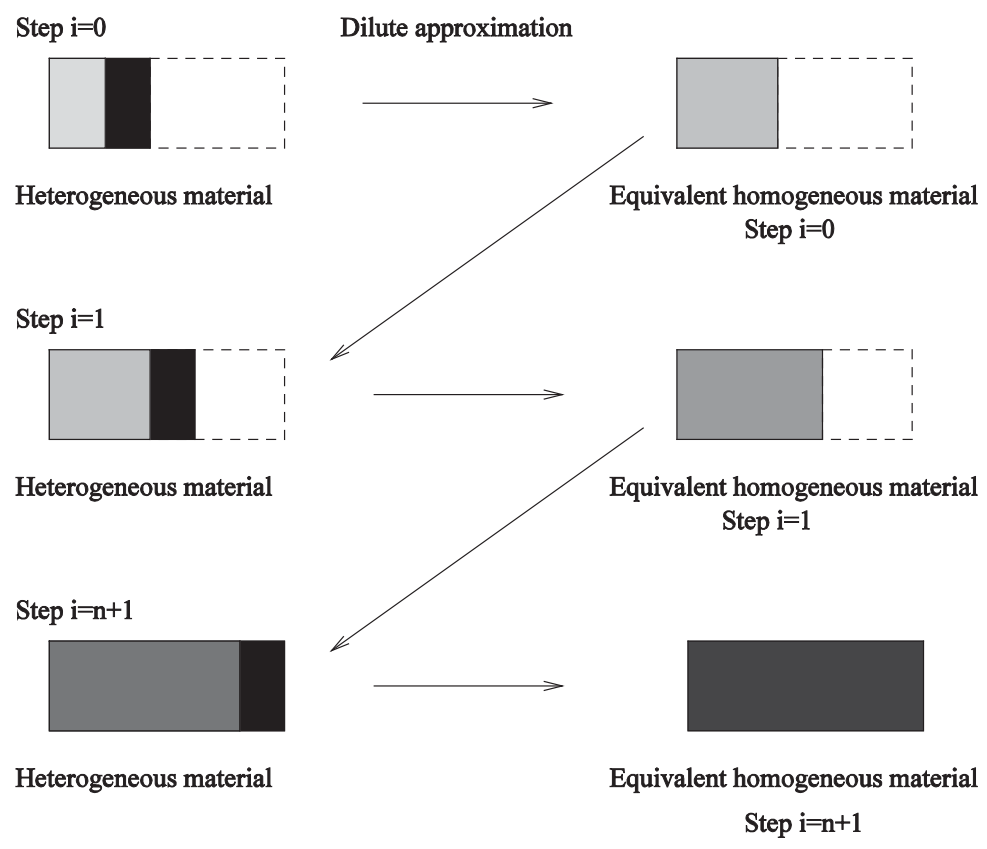

Fig. 3. Schematic illustration of the sequential method. The reinforcing material is added little by little; each addition is followed by a homogenization of the resulting composite; the procedure ends when the desired volume fraction is reached.

lying in the matrix. This three phase composite is virtually decomposed into an aggregate of two pseudo-grains: "matrix+voids" and "matrix+carbon". Each two-phase pseudo grain is homogenized (first step) and then the effective properties of the aggregate are computed (second step).

For the first step almost any homogenization scheme valid for two-phase composites with aligned inclusions can be used, e.g. Mori-Tanaka (M-T). Care must be taken when one of the phases consists actually of voids.

A way to achieve the second step is to assume the same deformation -- Voigt-like hypothesis -- inside both pseudo-grains. Other choices can be made (Friebel et al., 2006). One of them assumes the same strains in both matrix phases of the two pseudo-grains. Combined with $\mathrm{M}-\mathrm{T}$ at first step, the two-step approach degenerates then into the classical direct extension of the M-T scheme to multi-phase composites.

We draw attention to the fact that this two-step procedure can handle CNT composites with long nanotubes as well as short ones, aligned or not in the matrix. For composites with misaligned CNT extra pseudo-grains (in addition to the preexisting ones) are required, one for each orientation (Friebel et al., 2006).

The models used in the numerical simulations (section 4) are identified by the homogenization schemes used for the steps. They are labeled "two-step (M-T/Voigt)" and "two-step (M-T/M-T)". The latter is the direct extension of the M-T scheme to multi-phase composites. 


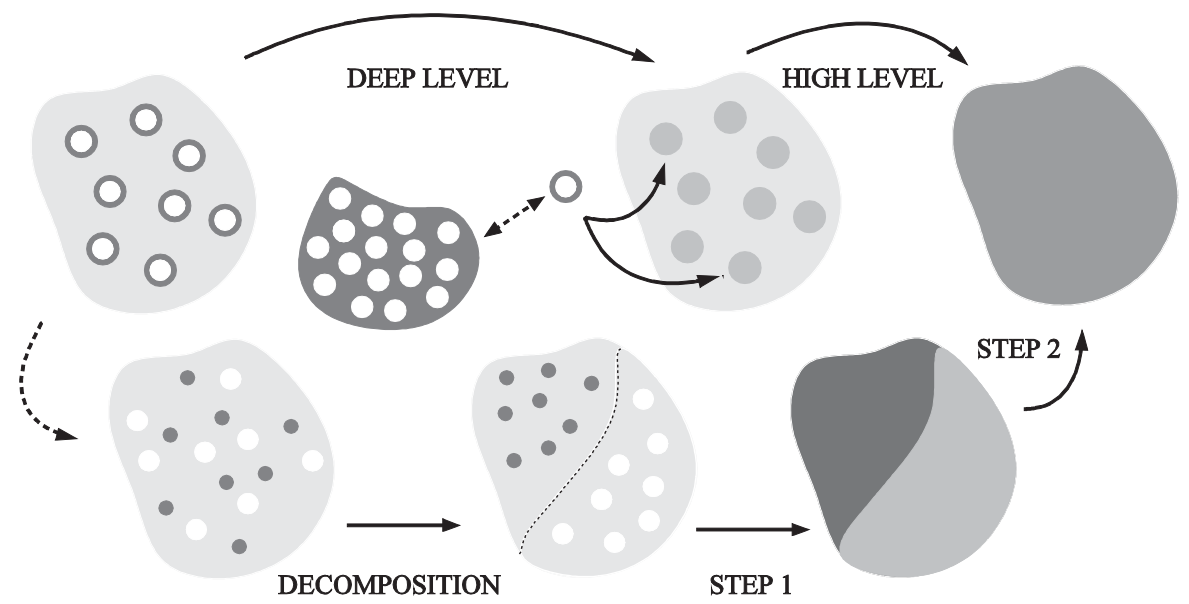

Fig. 4. Schematic view of the two-step (bottom) and two-level (top) homogenization procedures for the effective properties of SWNT composites. For each step/level a twophase homogenization model is required.

\subsection{The two-level procedure}

The two-level procedure was proposed by (Friebel et al., 2006) for coated inclusionreinforced materials. It is based on the idea that the matrix sees reinforcements (CNT) which are themselves composites (carbon+void). The methodology is illustrated on Fig. 4 (top) for composites with aligned CNT. Each CNT is seen (deep level) as a two-phase composite (carbon matrix with cavities) which, once homogenized, plays the role of a homogeneous reinforcement for the matrix material (high level).

A two-level recursive application of two-phase homogenization schemes (e.g. M-T) is thus proposed. As far as the choice of the two-phase models is concerned, the same remarks as for the first step of the two-step approach hold for the deep level. In addition, the inclusion phase is no more isotropic at high level.

Like the two-step approach, the two-level procedure is able to handle long as well as short CNT. The two-level procedure cannot be used stand-alone for composites with misaligned CNT. For this kind of materials, a combined two-step/two-level method is designed: the virtual decomposition is based only on the orientations; the pseudo-grains are homogenized (first step) using the two-level procedure; the second step is performed with the Voigt assumption.

A particular scheme is identified by the schemes used to perform the levels. In section 4, choosing M-T for both levels is labeled "two-level (M-T/M-T)". The same label is abusively used for composites with misaligned CNT, keeping in mind that it is indeed a combination of the two-level and two-steps procedures, as described above.

\subsection{D FE for hexagonal array arrangement}

The following FE procedure is only valid for composites with long and aligned CNT. We suppose a hexagonal array arrangement (Fig. 5) of the CNT in the matrix. As a result, the overall behavior is transversely isotropic. The macroscopic stiffness tensor is (partially) filled-in with help of only two generalized plane strain (2D) simulations. 
The unit-cell is shown on Fig. 5. It is reduced to a quarter of its size by exploiting two symmetry planes. Knowing the dimensions of the CNT (radii $R_{v}$ and $R_{c}$ on Fig. 5) and their volume fraction, one can compute the half tube inter-distance $\mathrm{d}$. The height and width of the reduced unit-cell are $\mathrm{h}=\mathrm{d}$ and $\mathrm{l}=\mathrm{d} \sqrt{3}$, respectively.

\begin{tabular}{|lll|}
\hline & Axial tension & Transverse tension \\
\hline $\mathrm{x}_{1}=0$ & $\mathrm{u}_{1}=0$ & $\mathrm{u}_{1}=0$ \\
$\mathrm{x}_{2}=0$ & $\mathrm{u}_{2}=0$ & $\mathrm{u}_{2}=0$ \\
$\mathrm{x}_{1}=1$ & $\mathrm{u}_{1}=$ constant & $\mathrm{u}_{1}=\mathrm{u}$ \\
$\mathrm{x}_{2}=\mathrm{h}$ & $\mathrm{u}_{2}=$ constant & $\mathrm{u}_{2}=$ constant \\
Reference node & $\mathrm{u}_{3}=\mathrm{u}$ & $\mathrm{u}_{3}=0$ \\
\hline
\end{tabular}

Table 1. Displacement boundary conditions for the transverse and axial tension tests. The unit-cell (Fig. 5) is in the $x_{1}-x_{2}$ plane, the symmetry planes are $x_{1}=0$ and $x_{2}=0$.

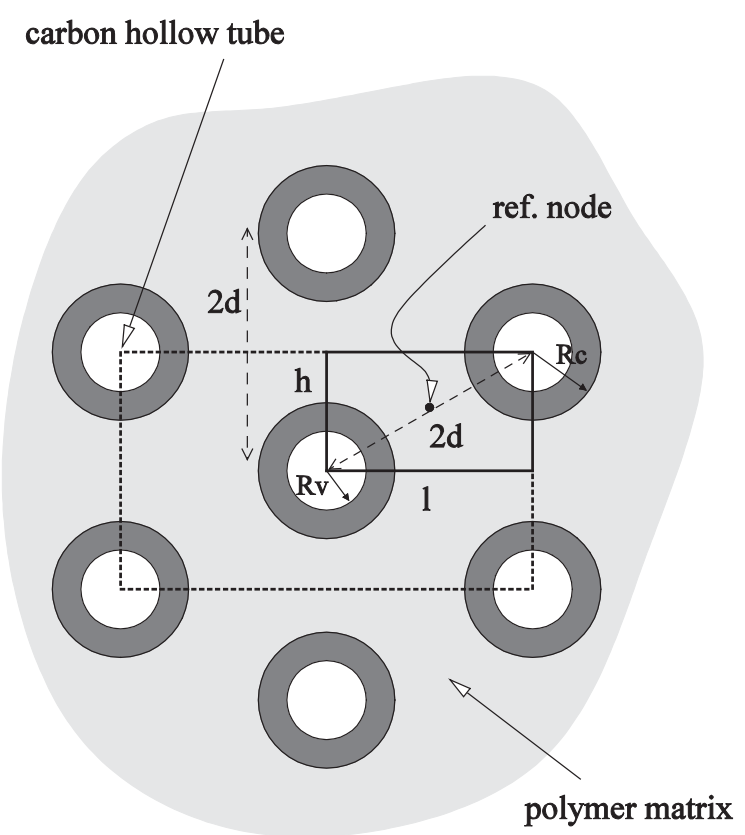

Fig. 5. Periodic hexagonal array arrangement of long carbon nanotubes inside the composite. Generalized plane strain (the reference node is shown) FE simulations are conducted on the two dimensional rectangular unit cell reduced to its quarter by symmetry.

Two tension tests are needed to obtain 4 among the 5 independent effective elastic moduli: a plane strain transverse tension followed by an axial tension under generalized plane strain conditions. Assuming the CNT are aligned along the third direction, the appropriate boundary conditions are summarized in Table 1. With this method, only the effective longitudianl shear modulus remains unknown. 


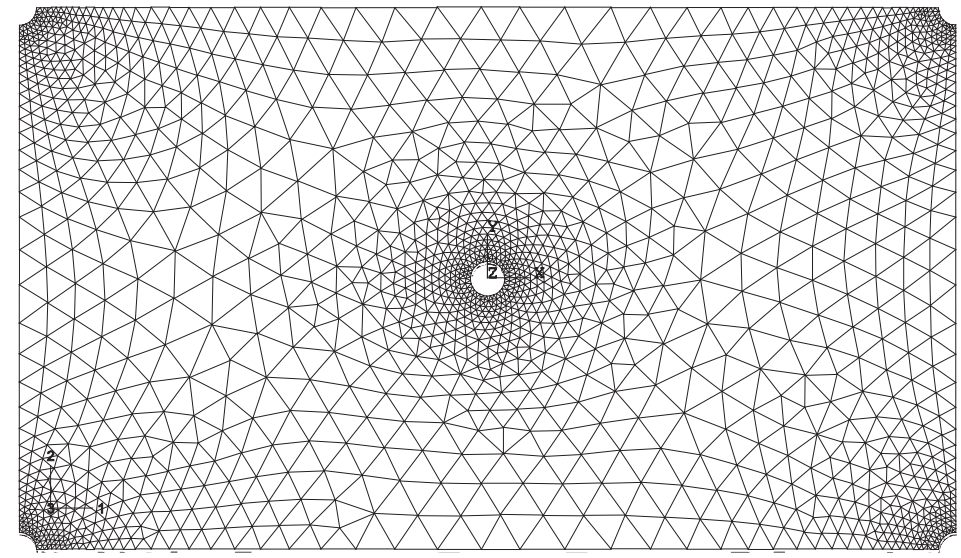

Fig. 6. Unit cell with periodic boundary conditions: 2D view of FE mesh corresponding to $10 \%$ of SWNT.

\subsection{D FE with periodic boundary conditions}

Again we consider composites with long and aligned SWNT and assume a hexagonal array arrangement. However, both the unit cell and the boundary conditions are different from those of section 3.4. Here, the unit cell is the large rectangle with dotted sides of Fig. 5, i.e. with one tube at its center and a quarter of a tube at each corner. Actually, a 3D cell with unit thickness is considered. Periodic boundary conditions are applied, as in (Segurado \& Llorca, 2002) for other micro-structures.

Periodic boundary conditions are written in a form representing periodic deformation and antiperiodic tractions on the boundary of the cell. The boundary conditions applied on an initially periodic cell perserve the periodicity of the cell in the deformed state. This model allows us to compute the whole mechanical properties of the transversely isotropic composite.

The selected cell is meshed using NETGEN (NETGEN, 2004) mesher, with which periodic boundary conditions can be applied. The FE meshes consisted of linear tetrahedral elements, Figs. 6 and 7 show respectively, a 2D and 3D view of the FE mesh corresponding to $10 \%$ of SWNT.

\section{Numerical predictions}

The models described above are used to predict the overall mechanical properties of various SWNT reinforced polymers. Our analytical schemes (sequential, two-step and two-level) are compared to FE calculations in section 4.1 for polyimide (LaRC-SI) and epoxy systems. Confrontation to experimental data is made in section 4.2 for a polypropylene matrix composite. Through all these composites, different orientations (fully aligned, random in space or in plane) and shapes (aspect ratio) of the CNT are simulated. In all figures, $\mathrm{R}_{\mathrm{V}}$ and $R_{c}$ represent the cylindrical cavity radius and the carbon coating radius, respectively (see Fig. 5). In all following figures, the nanotube volume fraction is the sum of cavities and carbon coating volume fractions. 


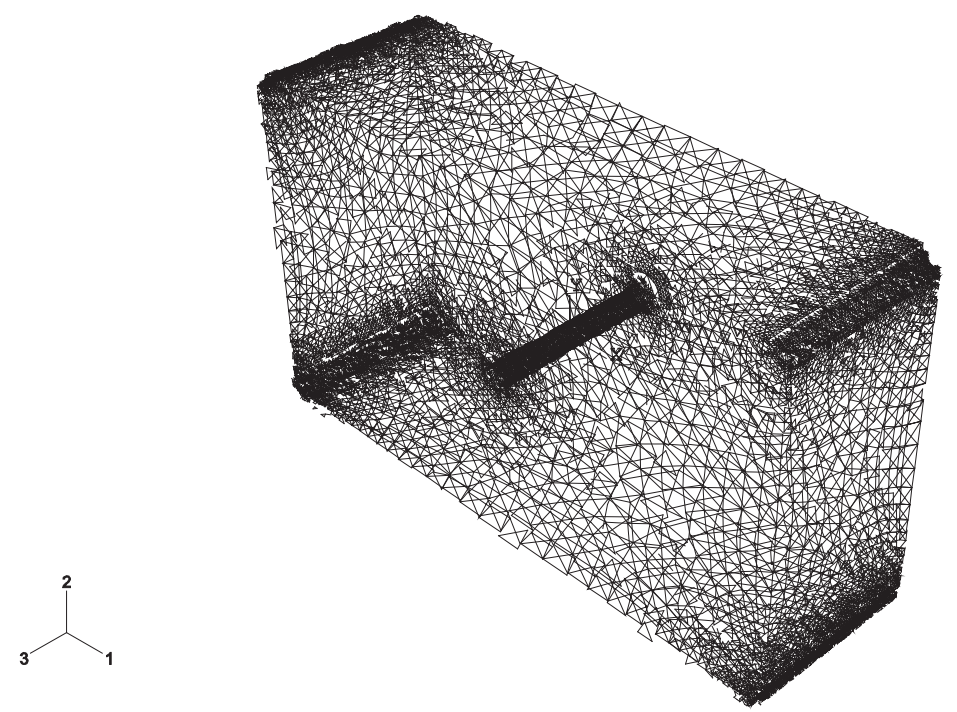

Fig. 7. Unit cell with periodic boundary conditions: 3D view of FE mesh corresponding to $10 \%$ of SWNT.

\subsection{Comparison between FE predictions and micromechanical models 4.1.1 Long SWNT/polyimide composite}

A polyimide (LaRC-SI) is reinforced with fully aligned, long homogeneously dispersed SWNT; see Table 2 for the properties. The predicted mechanical response of the composite relative to the mechanical properties of LaRC-SI is reported in Figs. 8a-8d.

\begin{tabular}{|l|l|l|}
\hline & Young's modulus (GPa) & Poisson's ratio \\
\hline LaRC-SI & 3.8 & 0.4 \\
\hline Continuumgraphene & 2520 & 0.25 \\
\hline
\end{tabular}

Table 2. Elastic constants of LaRC-SI and Continuum graphene, Young's modulus and

Poisson's ratio of Continuum graphene are calculated in section 2, those of LaRC-SI were found in (Odegard et al., 2003).

\section{Interpretation}

Comparing the two FE results, it is found that, the 3D FE calculations with periodic boundary conditions coincide with the 2D FE ones for all the long SWNT/Polyimide composite mechanical properties. The transverse shear modulus of the composite is found by the 3D FE simulations. As one can expect, all models give the same prediction of the longitudinal elastic modulus which indeed obeys to the rule of mixture. However for the transverse Young's modulus $\mathrm{E}_{\mathrm{T}}$ (Fig. 8a), the longitudinal shear modulus $\mathrm{G}_{\mathrm{L}}$ (Fig. 8a) and the transverse shear modulus $\mathrm{G}_{\mathrm{T}}$ (Fig. 8c), the sequential and the two-level (M-T/M-T) 
predictions are close to FE results, while the other models fail in a spectacular way. For the longitudinal Poisson's ratio $v_{L T}$ (Fig. 8d) -- for tension in the L-direction, $v_{L T}=\frac{\varepsilon_{T}}{\varepsilon_{L}}$, it is the two-level (M-T/M-T) model which gives the best predictions compared to the FE results. The "knee" in the transverse Young's modulus (Fig. 8a) shown by all homogenization methods at approximately $1 \%$ SWNT volume fraction is a-priori surprising. Thankfully it is confirmed by the FE model. This behavior is actually due to the high contrast between the moduli of the phases. From a homogenization scheme point of view the dependence of the transverse Young's modulus on the volume fraction of fillers is indeed typical to a MoriTanaka scheme for two-phase fibrous composites when the contrast between the rigidities becomes important.

Figs. 8a-8c exhibit a behavior of the two-step approach that has already been observed for viscoelastic coated fiber composites by (Friebel et al., 2006). Their numerical simulations show that using a two-step model for these composites may lead to erroneous estimates (compared to FE results) depending on the repartition of the materials between the phases. Our SWNT composite here modeled as a three phase material of this kind seems to fall into this category for which the two-step approach is completely inefficient.

The pretty good behavior of two-level already observed in (Friebel et al., 2006) is once again illustrated here (Figs. 8a-8d). Surprisingly, the sequential method which is alway close to the two-level (M-T,M-T) scheme on Figs. 8a-8c fails in the prediction of the longitudinal Poisson's ratio (Fig. 8). We don't have any explanation for this yet. This issue requires further investigations.

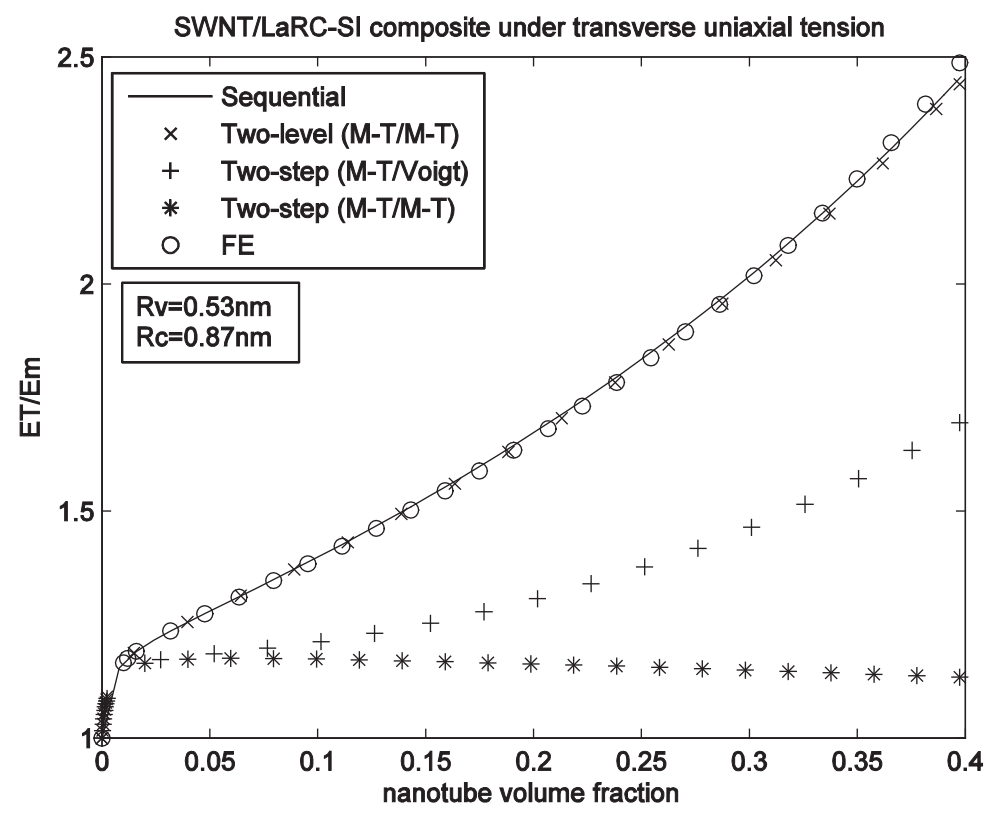

Fig. 8a. SWNT/LaRC-SI composite with long and aligned reinforcements. Normalized properties: comparison between the predictions of unit cell FE and micromechanical models. Transverse Young's modulus. 


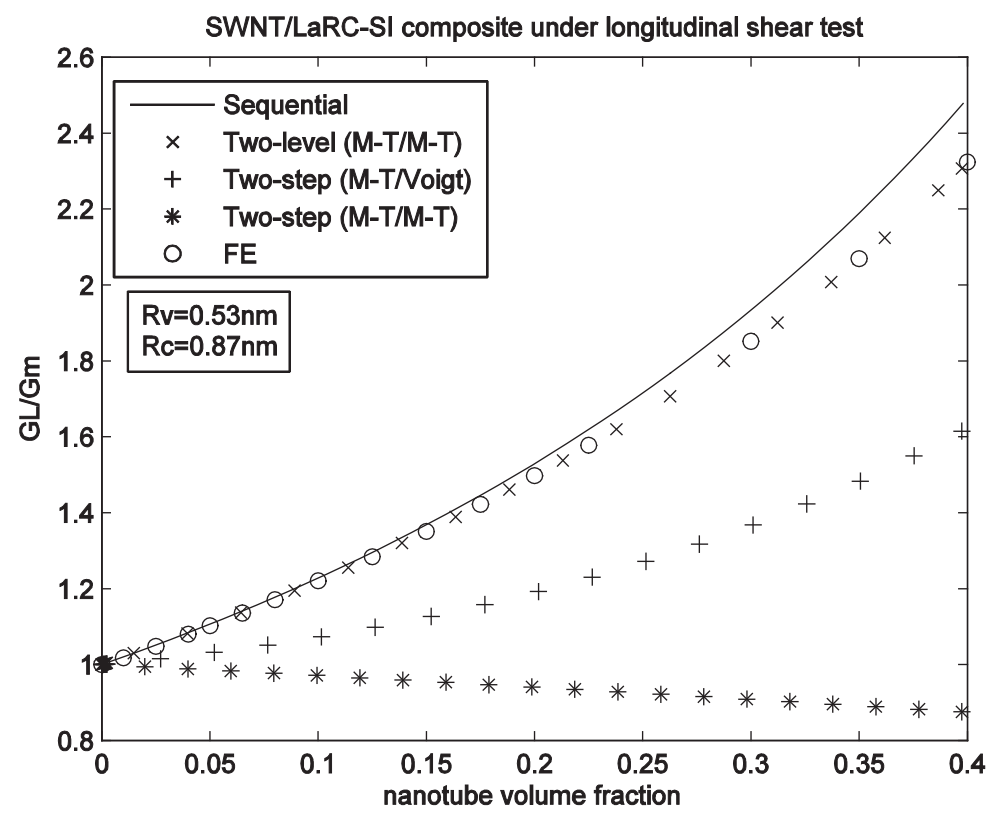

Fig. 8b. Same as Fig. 8a. Longitudinal shear modulus.

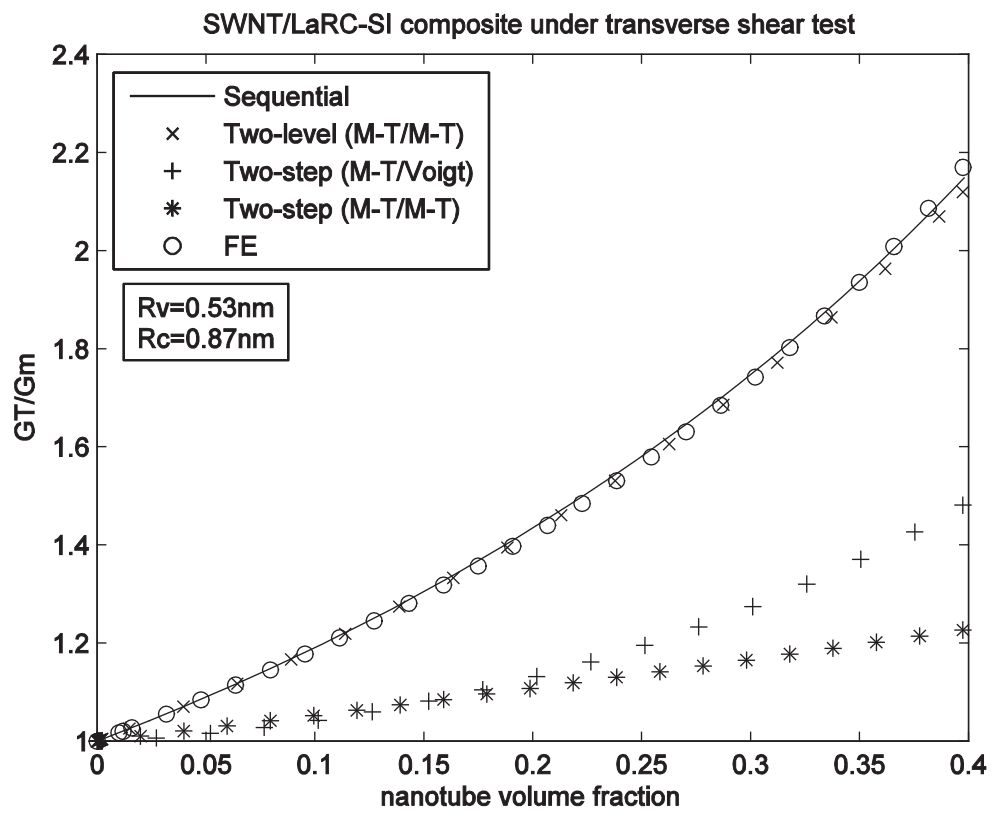

Fig. 8c. Same as Fig. 8a. Transverse shear modulus. 


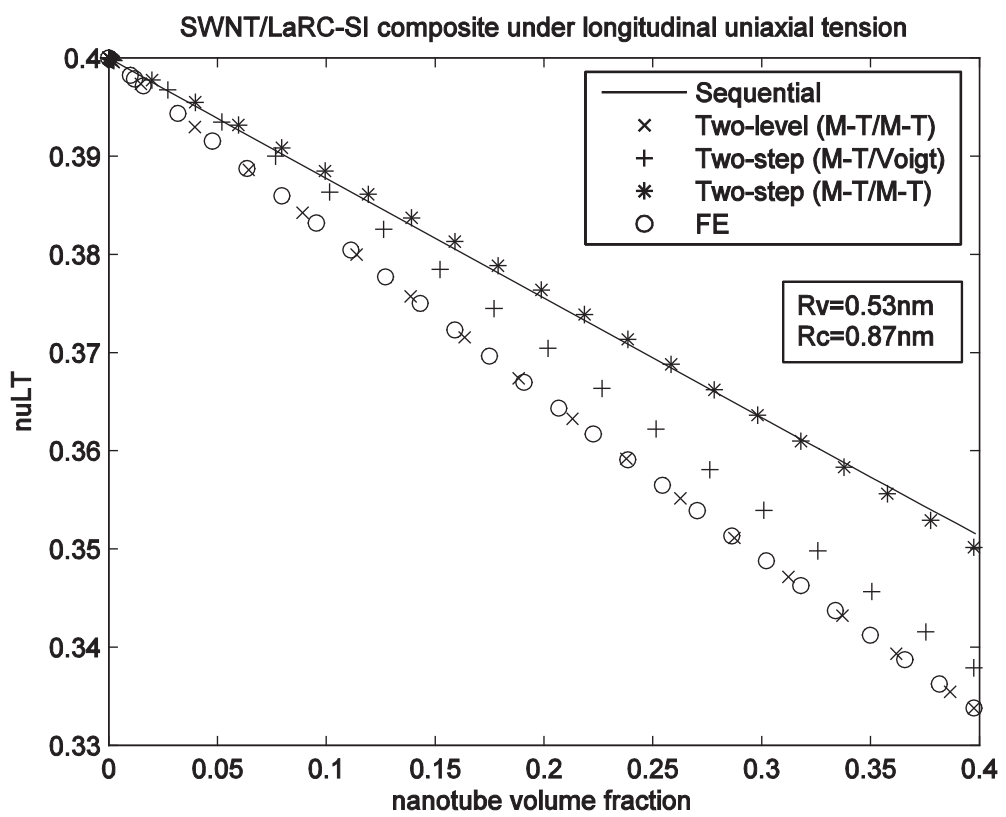

Fig. 8d. Same as Fig. 8a. Poisson's ratio $v_{\text {LT }}$

\subsubsection{SWNT/Epoxy composite}

The stiffness enhancement of an epoxy matrix comprising SWNTs is investigated here for three morpholigies namely 3D randomly oriented (Fig. 9a), 2D in-plane randomly oriented (Fig. 9b) and fully aligned reinforcements (Fig. 9c). On these figures our estimates of effective elastic moduli are compared with FE calculations after (Lusti \& Gusev, 2004).

The authors designed orthorhombic cells containing 50 equally sized and fully aligned nanotubes as well as cubics cells with 350 randomly oriented (3D or 2D planar) nanotubes distributed by employing a Monte Carlo algorithm. All geometries were meshed with tetrahedral elements - up to several millions elements were necessary in some cases - and periodic boundary conditions were applied. They conducted both direct calculations on the cubic cells with misaligned CNTs and orientation averaging of the moduli coming from orthorhombic cells. It is not clear which method was chosen to obtain their results given on Figs. 9b and 9c. But the authors report that a perfect match is obtained between both approaches for the 3D random orientation while deviations of up to $8 \%$ can be observed in the $2 \mathrm{D}$ case.

(Lusti \& Gusev, 2004) modeled the nanotubes as massive cylinders with hemispherical caps at both ends. The elastic properties they used in their two-phase modeling of the SWNT/Epoxy composites are reported in Table 3. We used the same material parameters but added a void phase, affecting the cylinders properties to the graphene coating phase. The volume ratio between void and graphene was obtained from the chirality of the SWNT reported in (Lusti \& Gusev, 2004) using a formula after (Toshiaki et al., 2004). 


\begin{tabular}{|l|l|l|}
\hline & Young's modulus $(\mathrm{GPa})$ & Poisson's ratio \\
\hline Epoxy & 3 & 0.35 \\
\hline SWNT & 1220 & 0.275 \\
\hline
\end{tabular}

Table 3. Elastic constants of epoxy and SWNT (modeled as massive cylinders), after (Lusti \& Gusev, 2004).

\section{Interpretation}

We conclude that all three composite morphologies have one property in common, namely that the overall effective Young's modulus increases linearly with the nanotube volume fraction for any aspect ratio in $[100, \infty[$.

For all three composite morphologies and for the two aspect ratios, the predictions given by the two-level (M-T/M-T), two-step (M-T/Voigt) and two-step (M-T/M-T) models are quite close to the FE data.

Furthermore, it appears that the stiffness enhancement that can be achieved with fully aligned, $2 \mathrm{D}$ random in-plane and 3D random orientation states differ considerably. For a composite comprising 12\% fully aligned SWNT (Fig. 9c) one can expect an enhancement by a factor 30-45 for the longitudinal Young's modulus $\mathrm{E}_{\mathrm{L}}$. A composite comprising 12\% 2D randomly oriented SWNT (Fig. 9b) the in-plane Young's modulus is increased by a factor of 11-15 whereas with 3D randomly oriented SWNT (Fig. 9a) the Young's modulus E is only raised by a factor of 6-9.

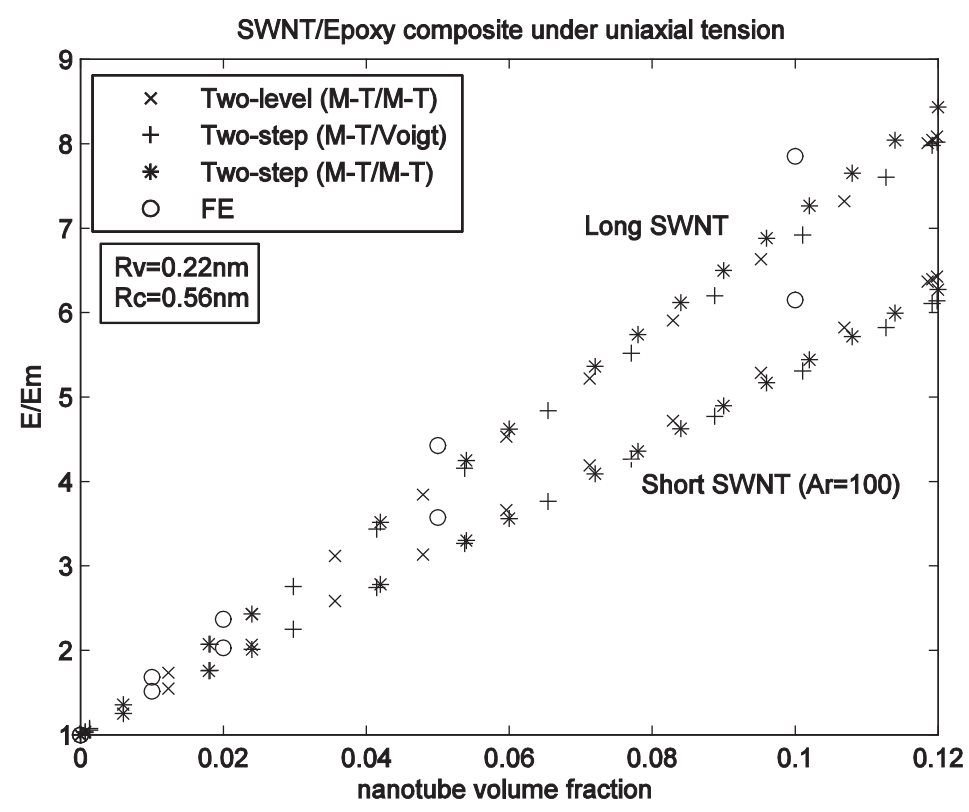

Fig. 9a. Epoxy composite with 3D randomly oriented reinforcements. Normalized Young's modulus: comparison between FE (Lusti \& Gusev, 2004) predictions and micromechanical models. 


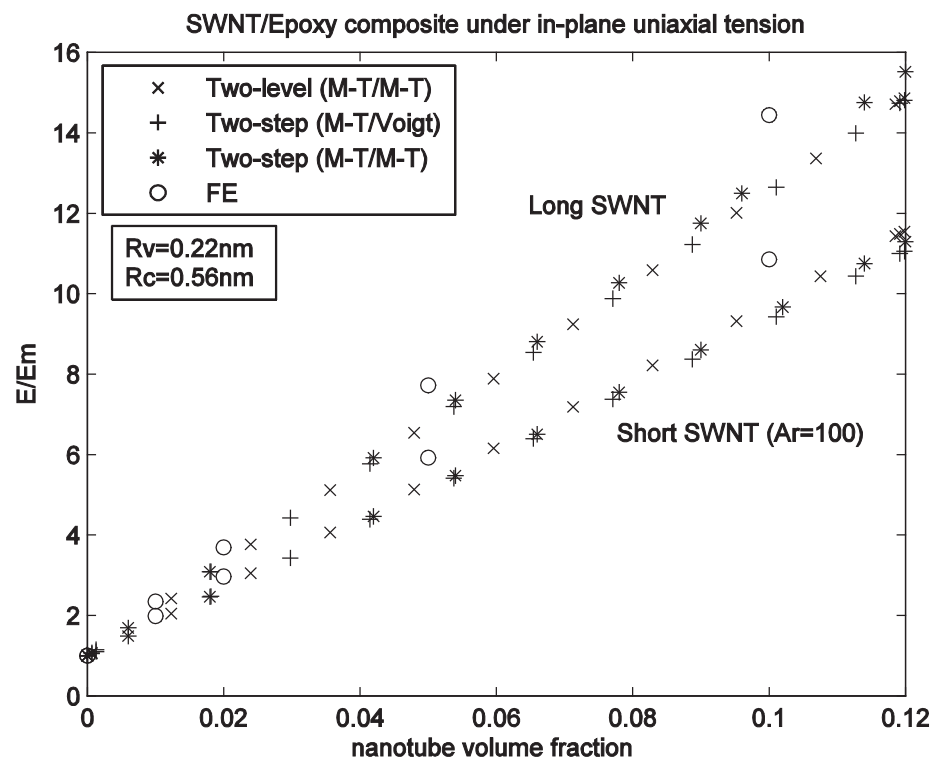

Fig. 9b. SWNT/Epoxy composite with 2D randomly oriented reinforcements. Normalized in-plane Young's modulus: comparison between FE (Lusti \& Gusev, 2004) predictions and micromechanical models.

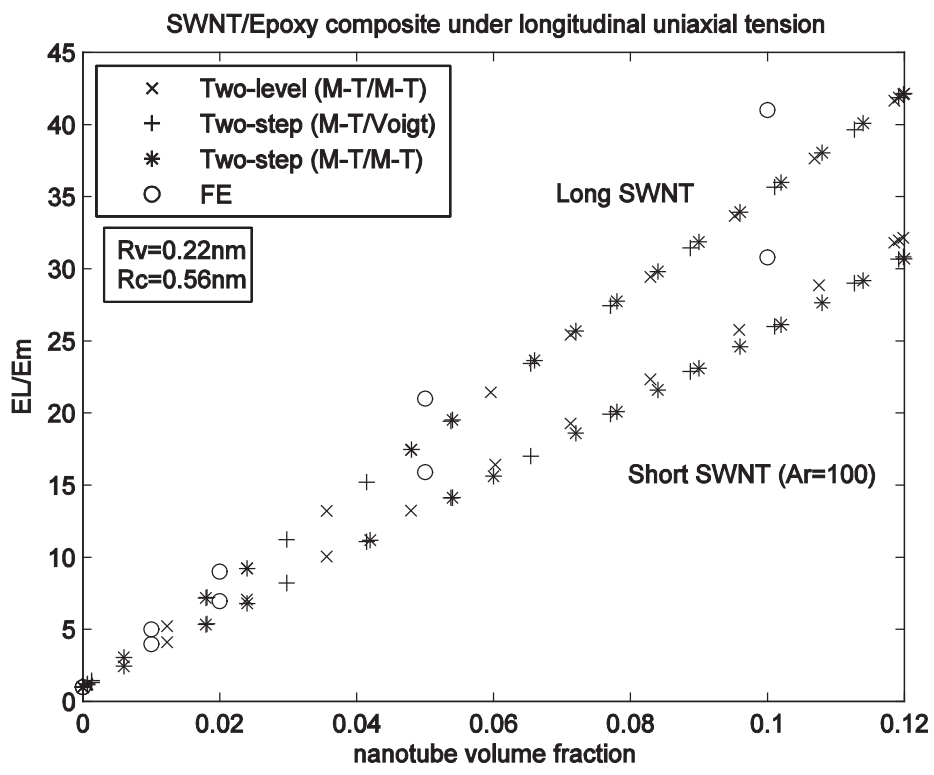

Fig. 9c. SWNT/Epoxy composite with fully aligned reinforcements. Normalized longitudinal Young's modulus: comparison between FE (Lusti \& Gusev, 2004) predictions and micromechanical models. 


\subsection{Comparisons between experimental data and micromechanical models}

The exceptional mechanical properties of SWNT make them good reinforcements even at very low concentrations. The reinforcement of polymers and other matrix systems with SWNT has previously been demonstrated to increase the physical properties of the matrix materials. Such studies include SWNT/Polypropylene (Valentini et al., 2003).

\subsubsection{SWNT/Polypropylene composite}

In our study a SWNT concentration ranging from $0 \%$ to $0.48 \%$ as SWNT volume fractions incorporated in the polypropylene is investigated; see Table 4 for the properties. Young's modulus and Poisson's ratio of 3D randomly oriented SWNT/Polypropylene composite with various SWNT volume fractions and for an aspect ratio equal to 100 are reported in Figs. 10a and 10b.

\begin{tabular}{|l|l|l|}
\hline & Young's modulus $(\mathrm{GPa})$ & Poisson's ratio \\
\hline Polypropylene & 0.855 & 0.43 \\
\hline Continuum graphene & 2550 & 0.25 \\
\hline
\end{tabular}

Table 4. Elastic constants of polypropylene and Continuum graphene. Young's modulus and Poisson's ratio of Continuum graphene are calculated in section 2, those of polypropylene were found in (Lopez et al., 2005).

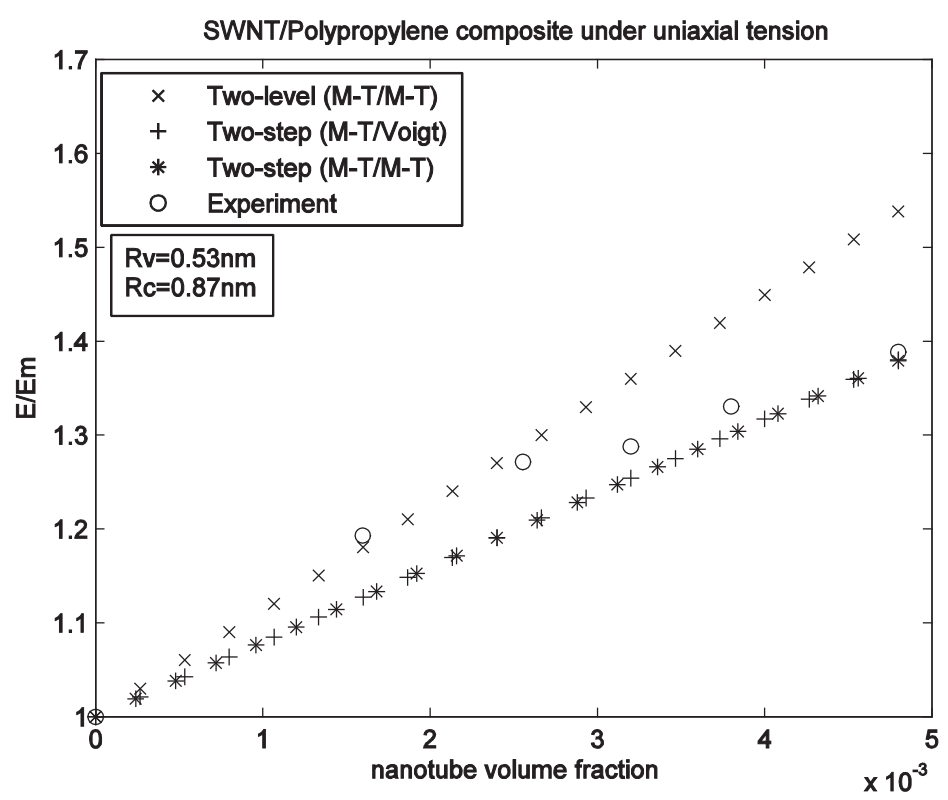

Fig. 10a. SWNT/Polypropylene composite with 3D randomly oriented reinforcements for an aspect ratio $\mathrm{Ar}=100$. A comparison between experimental data after (Lopez et al., 2005) and micromechanical models. Normalized Young's modulus. 


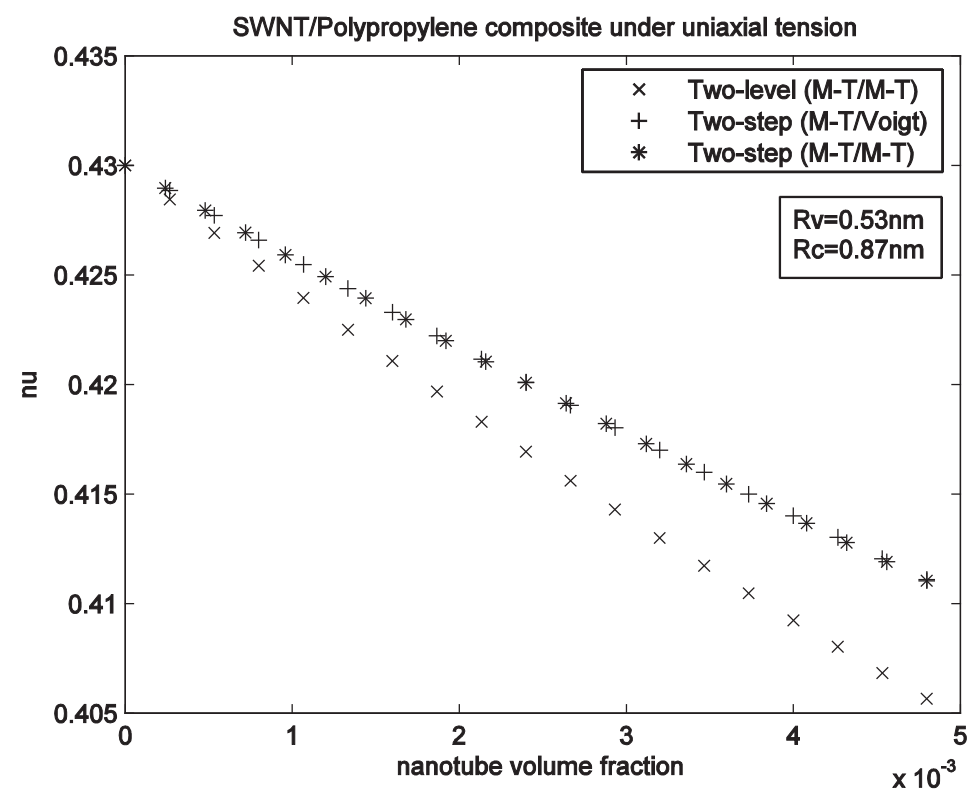

Fig. 10b. Same as Fig. 10a. Poisson's ratio.

\section{Interpretation}

From these results, it is deduced that the reinforcing effect of SWNT is very important and as the SWNT content in the polymer increases, the Young's modulus increases rapidly. For a polypropylene comprising $0.48 \%$ of SWNT, the Young's modulus E is increased by a factor of 1.4 but the Poisson's ratio decreases by a factor of 1.06 .

Our results are compared with experimental results given by (Lopez et al., 2005). The nonlinear behavior in the experimental data (Fig. 10a) may be due to a change in the microstructure with respect to the concentration of SWNTs. This is observed experimentally (Lopez et al., 2005) and characterized by the forming of nanotubes aggregates and a modification of the matrix' crystallinity. Neither the clustering nor the modification of the constituents' material properties is taken into account in our models. It is thus not surprising to see differences in the behavior of measurements and numerical predictions as the volume fraction of SWNTs increases. All numerical models however give estimates of Young's modulus with the right order of magnitude over the whole range of concentrations. For low content of SWNT, where the modeling assumptions on the microstructure are valid, the two-level method performs better that the two-step one.

The Poisson's ratio predictions delivered by the two-level (M-T/M-T), the two-step (M$\mathrm{T} / \mathrm{M}-\mathrm{T}$ ) and the two-step (M-T/Voigt) models are reported in Fig. 10b. We remark that the two-step (M-T/M-T) and the two-step (M-T/Voigt) models deliver the same prediction for the Poisson's ratio.

\subsubsection{SWNT/PVA composite}

In this section a $2.89 \% \mathrm{SWNT}\left(\mathrm{R}_{\mathrm{v}}=0.33 \mathrm{~nm}, \mathrm{R}_{\mathrm{c}}=0.67 \mathrm{~nm}\right)$ volume fraction incorporated in the polyvinyl alcohol (PVA) is investigated; see Table 5 for the properties. Young's modulus and 
Poisson's ratio of 3D randomly oriented SWNT/PVA composite with long reinforcements are reported in Table 6 .

\begin{tabular}{|l|l|l|}
\hline & Young's modulus $(\mathrm{GPa})$ & Poisson's ratio \\
\hline PVA & 25.6 & 0.4 \\
\hline SWNT & 2550 & 0.25 \\
\hline
\end{tabular}

Table 5. Elastic constants of PVA and Continuum graphene. Young's modulus and Poisson's ratio of Continuum graphene are calculated in section 2, those of PVA were found in (Zhang et al., 2004).

\begin{tabular}{|l|l|l|}
\hline & Young's modulus (GPa) & Poisson's ratio \\
\hline Experiment & 35.8 & -- \\
\hline Two-level (M-T/M-T) & 36.13 & 0.378 \\
\hline Two-step (M-T/Voigt) & 35.51 & 0.376 \\
\hline Two-step (M-T/M-T) & 35.76 & 0.375 \\
\hline
\end{tabular}

Table 6. SWNT/PVA composite with 3D randomly oriented and long reinforcements. A comparison between experimental data after (Zhang et al., 2004) and micromechanical models for $2.89 \%$ SWNT volume fraction.

\section{Interpretation}

From Table 6, it is seen that the modulus of the composite is $40 \%$ higher than that of PVA which is it self 8 times stiffer than that of the other studied matrix materials.

Our results were compared with experimental results given by (Zhang et al., 2004).

For $2.89 \%$ SWNT volume fraction, Young's modulus predictions delivered by all models are consistent with the experiment data.

The same Table 6 shows that the Poisson's ratio predictions delivered by all schemes are also very close.

\section{Conclusion}

After the characterization of the discrete nanotube structure using a homogenization method based on energy equivalence, the sequential, the two-step (M-T/M-T), the two-step (M-T/Voigt), the two-level (M-T/M-T) and finite element models were used to predict the elastic properties of SWNT/Polymer composites. The data delivered by the micromechanical models are compared against those obtained by finite element analyzes or experiments. For fully aligned, long nanotube polymer composite, it is the sequential and the two-level (M-T/M-T) models which delivered good predictions. For all composite morphologies (fully aligned, two-dimensional in-plane random orientation, and threedimensional random orientations), it is the two-level (M-T/M-T) model which gave good predictions compared to finite element and experimental results in most situations. There are cases where other micromechanical models failed in a spectacular way. 


\section{References}

Bai, JB. \& Ci, L. (2006). The reinforcement role of carbon nanotubes in epoxy composites with different matrix stiffness. Composite Science and Technology Vol. 66, pp. 599603.

Ben Hamida, A. \& Dumontet, H. (2003). Etude micromécanique du comportement de matériaux hétérogènes par une approche itérative. Proceedings of 6ème Colloque National en Calcul des Structures, Giens (France).

CASTEM 2000. A finite element software. $\backslash$ hfill $\backslash \operatorname{mbox}\{\}$ www.cines.fr/textes/cal-parallele/castem.html.

Cornell, W.D.; Cieplak, P.; Bayly, C.I.; Gould, I.R.; Merz, K.M.; Ferguson, D.M.; Spellmeyer, D.C.; Fox, T.; Caldell, J.W. \& Kollmann, P.A. (1995). A second generation force field for the simulation of proteins, nucleic acids, and organic molecules. Journal of the American Chemical Society Vol. 117, pp. 5179-5197.

Desprès, J.F., Daguerre, E. \& Lafdi, K. (1995). Flexibility of graphene layers in carbon nanotubes. Carbon Vol. 33, No. 1, pp. 87-92.

Fisher, F.T.; Bradshaw, R.D. \& Brinson, L.C. (2002). Effect of nanotube waviness on the modulus of nanotube-reinforced polymers. Applied Physics Letters Vol. 80, No. 24, pp. 4647.

Friebel, C.; Doghri, I. \& Legat, V., (2006). General mean-field homogenization schemes for viscoelastic composites containing multiple phases of coated inclusions. International Journal of Solids and Structures Vol. 43, No. 9, pp. 2513-2541.

Krishnan, A.; Dujardin, E.; Ebbesen, T.W.; Yianilos, P.N. \& Treacy, M.M.J. (1998). Measurement of the young's modulus of single-shell nanotubes using TEM. Physical Review B Vol. 58, No. 20, pp. 14013-14019.

Lopez Manchado, M.A.; Valentini, L.; Biagiotti, J. \& kenny, J.M. (2005). Thermal and mechanical properties of single-walled carbon nanotubes-polypropylene composites prepared by melt processing. Carbon Vol. 43, No. 7, pp. 1499-1505.

Lourie, O. \& Wagner, H.D. (1998). Evaluation of Young's modulus of carbone nanotubes by micro-Raman spectroscopy. Journal of Material Research Vol. 13, No. 9, pp. 24182422.

Lusti, H.R. \& Gusev, A.A. (2004). Finite element predictions for the thermoelastic properties of nanotube reinforced polymers. Modelling and Simulation in Materials Science and Engineering Vol. 12, No. 3, pp. 107-119.

NETGEN. An automatic three-dimensional tetrahedral mesh generator. $\backslash$ hfill $\backslash \operatorname{mbox}\{\}$ Joachim Schaberl, Johannes Kepler University, Linz, Austria, 2004.

Odegard, G.M.; Gates, T.S.; Wise, K.E. \& Nicholson, L.M. (2002). Equivalent-continuum modeling of nano-structured materials. Composite Science and Technology Vol. 62, pp. 1869-1880.

Odegard, G.M.; Gates, T.S.; Wise, K.E.; Park, C. \& Siochi, E.J. (2003). Constitutive modeling of nanotube-reinforced polymer composites. Composite Science and Technology Vol. 63, pp. 1671-1681.

Segurado, J. \& Llorca, J. (2002). A numerical approximation to the elastic properties of shpere-reinforced composites. Journal of the Mechanics and Physics of Solids, Vol. 50, No. 10, pp. 2107-2121.

Tijima, S. 1991. Helical Microtubules of Graphitic Carbon. Nature (London) 354, 56-58. 
Toshiaki, N., Kriengkamol T. \& Morinobu E., (2004). Prediction of elastic properties for single-walled carbon nanotubes. Carbon Vol. 42, pp. 39-45.

Zhang, X.; Liu, T.; Sreekumar, T.V.; Kumar, S.; Hu, X. \& Smith, K. (2004). Gel spinning of PVA/SWNT composite fiber. Polymer Vol. 45, pp. 8801-8807.

Valentini, L.; Biagiotti, J.; kenny, J.M. \& Santucci, S. (2003). Morphological characterization of single-wall carbon nanotube/polypropylene composites. Composites Science and Technology Vol. 63, No. 8, pp. 1149-1153. 


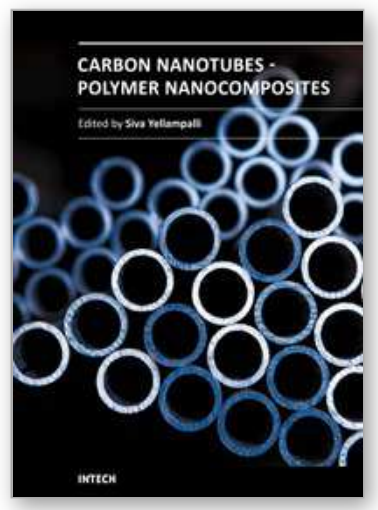

\section{Carbon Nanotubes - Polymer Nanocomposites}

Edited by Dr. Siva Yellampalli

ISBN 978-953-307-498-6

Hard cover, 396 pages

Publisher InTech

Published online 17, August, 2011

Published in print edition August, 2011

Polymer nanocomposites are a class of material with a great deal of promise for potential applications in various industries ranging from construction to aerospace. The main difference between polymeric nanocomposites and conventional composites is the filler that is being used for reinforcement. In the nanocomposites the reinforcement is on the order of nanometer that leads to a very different final macroscopic property. Due to this unique feature polymeric nanocomposites have been studied exclusively in the last decade using various nanofillers such as minerals, sheets or fibers. This books focuses on the preparation and property analysis of polymer nanocomposites with CNTs (fibers) as nano fillers. The book has been divided into three sections. The first section deals with fabrication and property analysis of new carbon nanotube structures. The second section deals with preparation and characterization of polymer composites with CNTs followed by the various applications of polymers with CNTs in the third section.

\section{How to reference}

In order to correctly reference this scholarly work, feel free to copy and paste the following:

Selmi Abdellatif and Hassis Hédi (2011). Prediction of the Elastic Properties of Single Walled Carbon Nanotube Reinforced Polymers: A Comparative Study of Several Micromechanical Models, Carbon Nanotubes - Polymer Nanocomposites, Dr. Siva Yellampalli (Ed.), ISBN: 978-953-307-498-6, InTech, Available from:

http://www.intechopen.com/books/carbon-nanotubes-polymer-nanocomposites/prediction-of-the-elasticproperties-of-single-walled-carbon-nanotube-reinforced-polymers-a-comparat

\section{INTECH}

open science | open minds

\section{InTech Europe}

University Campus STeP Ri

Slavka Krautzeka 83/A

51000 Rijeka, Croatia

Phone: +385 (51) 770447

Fax: +385 (51) 686166

www.intechopen.com

\section{InTech China}

Unit 405, Office Block, Hotel Equatorial Shanghai

No.65, Yan An Road (West), Shanghai, 200040, China

中国上海市延安西路65号上海国际贵都大饭店办公楼 405 单元

Phone: +86-21-62489820

Fax: $+86-21-62489821$ 
(C) 2011 The Author(s). Licensee IntechOpen. This chapter is distributed under the terms of the Creative Commons Attribution-NonCommercialShareAlike-3.0 License, which permits use, distribution and reproduction for non-commercial purposes, provided the original is properly cited and derivative works building on this content are distributed under the same license. 